\title{
Standard Model physics at the LHC
}

\author{
Jan Kretzschmar \\ University of Liverpool
}

\begin{abstract}
In this contribution to the volume "From My Vast Repertoire - The Legacy of Guido Altarelli" I discuss selected topics of Standard Model (SM) physics at the LHC and their connection to the work of Guido Altarelli. An overarching connection is given by Guido's fundamental contributions to our understanding of the strong force, parton distribution functions and their evolution, and techniques to calculate theoretical predictions through perturbative Quantum Chromodynamics. The increasingly precise LHC measurements of diverse processes can be confronted with SM predictions relying on these foundations to test the SM and contribute to the knowledge of the proton structure. The LHC experiments have also started to perform precise measurements of SM parameters, such as the mass of the $W$ boson, and have measured a variety of processes sensitive to interactions of multiple electroweak bosons. The discovery of the Higgs boson in 2012 and the measurements of its properties are crucial to establish the nature of the mechanism of electroweak symmetry breaking and the status of these studies is summarised.
\end{abstract}

\section{Introduction}

Before the year 2009, the start of the Large Hadron Collider (LHC) was eagerly anticipated in the whole particle physics community. As Guido Altarelli put it: "physics [was] in a deadlock and [needed] to be restarted." [1]. The Standard Model of particle physics (SM) was by many seen just a low-energy effective version of the ultimate theory. The LHC was expected to take our understanding to the next level. In the following I attempt to discuss where the endeavour to explore the SM at the energy frontier has lead us in the last seven years.

The LHC [2] is the most powerful accelerator ever built. From 2010 to 2012 it provided proton-proton $(p p)$ collisions at an unprecedented centre-of-mass energy of $\sqrt{s}=7-8 \mathrm{TeV}$ in Run 1. In 2015 the LHC started the Run 2 operation at $\sqrt{s}=13 \mathrm{TeV}$, close to the design value of $14 \mathrm{TeV}$. With this, it exceeds the typical energy scale of collisions at the previous generation of colliders, LEP, HERA and TeVatron, by about an order of magnitude. The LHC now regularly delivers an instantaneous luminosity higher by $50 \%$ than the original design goal of $10^{34} \mathrm{~cm}^{-1} \mathrm{~s}^{-1}$ to the ATLAS and CMS experiments [3, 4], exceeding previous colliders by about two orders of magnitude.

The SM describes the interactions of three generations of quarks and leptons through the gauge fields of the electroweak and strong interactions, with the electroweak symmetry being spontaneously broken by the Englert-Brout-Higgs mechanism. The unprecedented collision energy and large data sets provided by the LHC allow to probe the fundamental building blocks of matter and their interactions at a new level. It was certainly one of the "major physics discoveries of our time" [5] when the LHC experiments discovered "a" Higgs boson 
in 2012 [6, 7]. The Higgs boson was the final of the SM particles and was seen as "directly related to most of the major open problems of particle physics" [1].

It is important to remember, that the $p p$ collisions delivered by the LHC are complex interactions of composite, strongly-interacting particles. Without the framework provided by the SM, we would hardly be in a situation to exploit the data at such a detailed level as we do it these days: decades of efforts in theory and phenomenology enable us to connect the basic Lagrangian to Feynman diagrams and those to quantitative predictions of cross sections and decay rates. A striking feature of the LHC data is the vast spectrum of measurements (and searches) that are possible, thereby testing a wide diversity of processes as well as very different energy scales.

\section{The role of QCD in LHC physics}

The protons collided by the LHC are strongly-interacting particles composed of quarks and gluons. It is thus somewhat obvious, that the theory of strong interactions, Quantum Chromodynamics (QCD), is of the highest importance for LHC physics. This is also true, if the aim is to study electroweak interactions. Or, as Guido Altarelli put it: "the understanding QCD processes is an essential prerequisite for all possible discoveries" at the LHC [8]. Thanks to the work of Guido Altarelli and many others at the end of the 1970s, QCD was established as the theory of strong interactions and the framework of perturbative calculations was worked out. At the level of the Lagrangian "QCD is a 'simple' theory, but actually this simple theory has an extremely rich dynamical content" 8 .

The calculation of a cross section can be factorized into a part describing the structure of hadrons in terms of the Parton Distribution Functions (PDFs) and another part describing the interactions of the fundamental quarks and gluons. The PDFs are not static objects, but evolve with the energy scale of the interaction, a property studied by Dokshitzer, Gribov and Lipatov 19, 10, 11, and then rederived by Altarelli and Parisi "in a direct way from the basic vertices of QCD" 12. The resulting evolution equations are known as DGLAP equations and form a cornerstone of our understanding of QCD. The formulation given by Guido Altarelli has allowed to systematically improve the precision. The splitting functions that describe the evolution are nowadays known to the three-loop level [13, 14] and partially beyond this.

The elementary cross sections can be systematically calculated and similarly improved by an expansion in the strong coupling constant $\alpha_{\mathrm{S}}$. Guido Altarelli contributed to one of the first higher order calculations [15]: the production of di-leptons in hadron collisions through the Drell-Yan process [16]. The Drell-Yan process will be further discussed in Section 6. The mechanism to improve the calculations by considering diagrams higher-order in $\alpha_{\mathrm{S}}$ has been critical. For LHC physics purposes it has turned out, that the next-to-leading order (NLO) is the minimum to have a reliable prediction, but often the next-to-next-to-leading order (NNLO) still provides an important correction.

Guido Altarelli's contributions have such paved the way to quantitative predictions for QCD processes. The exploitation of the large LHC data sets keeps increasing the demands of experimentalists on the theory accuracy, which typically reaches the $5-10 \%$ percent level and may reach below $1 \%$ for simple processes. Still, the precision in the measurements is often better than the theory uncertainties, limiting the interpretation of the data. 


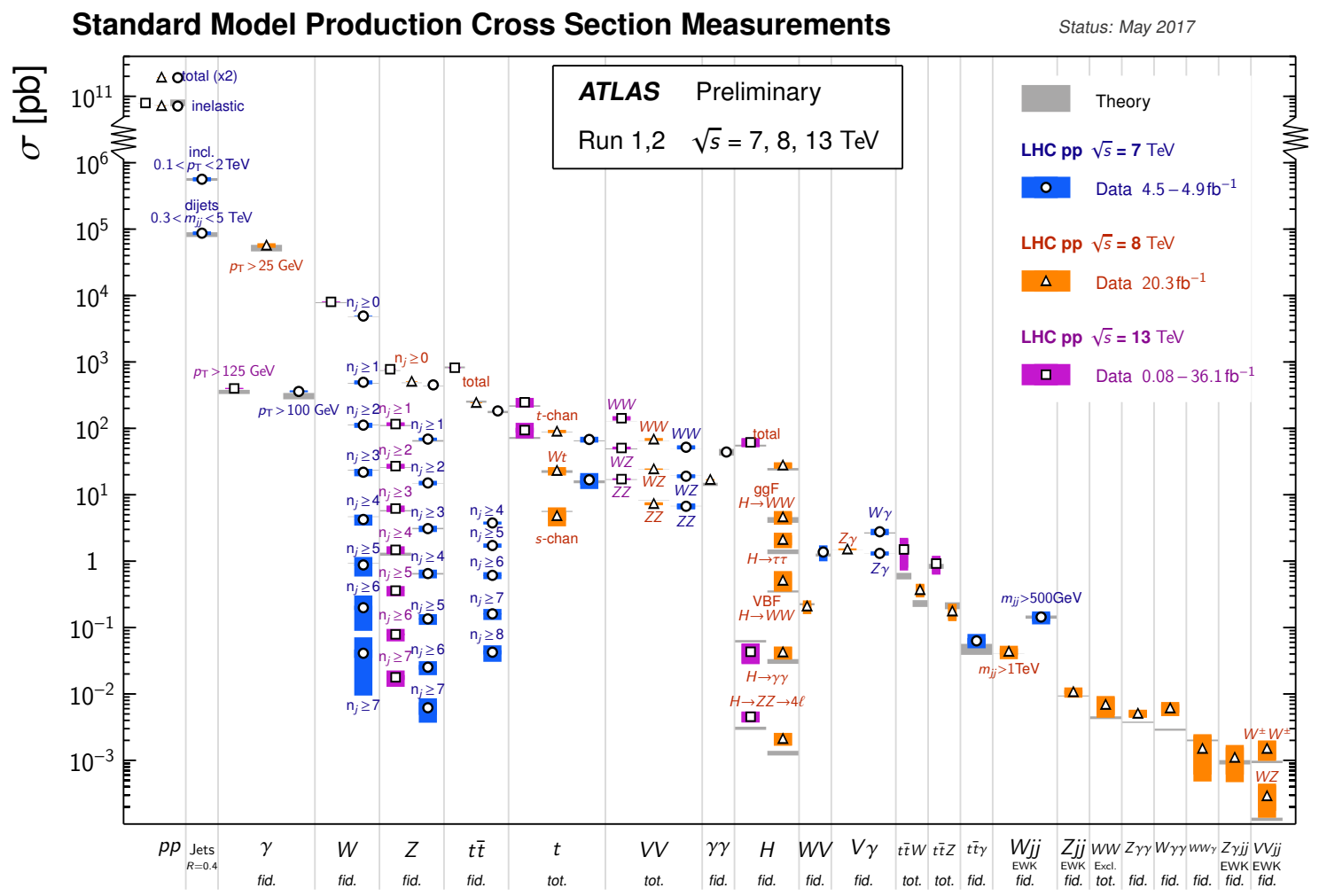

Figure 1: A summary of cross-section measurements in $p p$ collisions at $\sqrt{s}=7,8,13 \mathrm{TeV}$ for a variety of SM processes by the ATLAS collaboration [19]. The measurements are compared to the respective SM theory predictions. A similar summary is available for results by the CMS collaboration [20].

\section{A broad overview of SM processes studied at the LHC}

The diversity of processes and energy scales accessible at the LHC is exceptionally wide. A very much simplified summary of cross-section measurements is given in Fig. 1, where each measured point often stands for a complex, multi-differential analysis and typically 10-1000 differential measurements. The range of cross sections studied ranges from $100 \mathrm{mb}$ for the total $p p$ cross section to $1 \mathrm{fb}$ for the rarest processes, thus spanning 14 orders of magnitude. 1

The theoretical predictions for the vast majority of the processes displayed in Fig. 1 use the techniques of perturbative QCD. The necessary PDF information is largely derived from deep-inelastic lepton-nucleon scattering (DIS), although more recently the information from LHC measurements have started to become useful to constrain PDFs further. Fig. 2(left) illustrates the relevant range of the Björken- $x$ momentum fraction for LHC physics, which is roughly from $10^{-4}$ to 1 . Indeed DIS fixed-target experiments and the HERA ep collider cover this range, however a significant evolution in energy scale is required to reach the LHC region.

\footnotetext{
${ }^{1}$ In addition, this overview does not even attempt to capture many other important SM measurements performed with LHC data, as for example the study of rare or CP-violating B-hadron decays with the LHCb [17], CMS and ATLAS experiments or the study of Heavy Ion collisions and the quark-gluon plasma, where the major contributions of the ALICE experiments lie [18].
} 

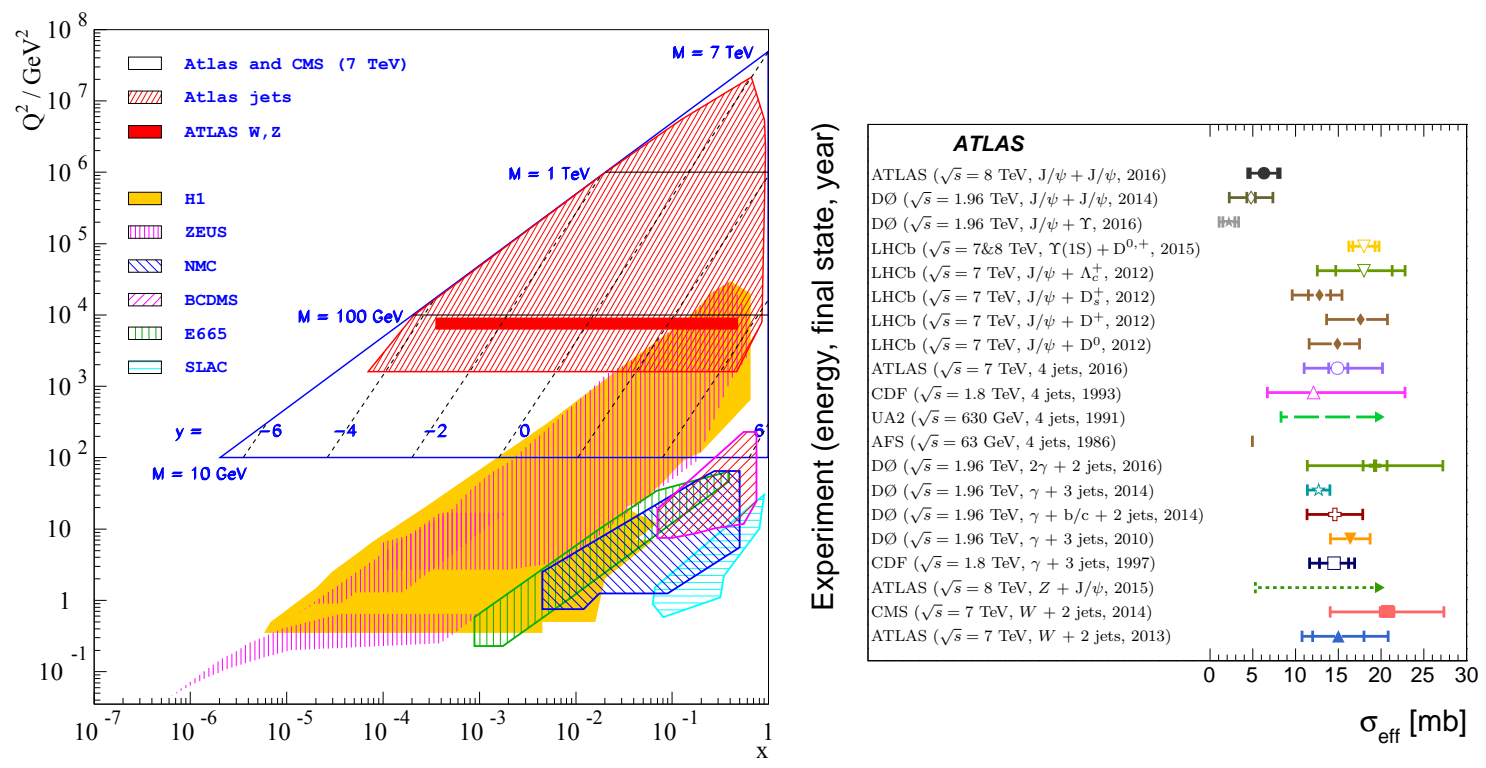

Figure 2: (left) Illustration of the plane in Björken- $x$ and scale $Q^{2}$ relevant for the determination of PDFs from DIS data by the HERA experiments (H1, ZEUS) and a selection of fixed-target experiments (NMC, BCDMS, E665, SLAC). The kinematic region accessed by the LHC in terms of mass $M$ and rapidity $y$ of the produced system is shown for $\sqrt{s}=7 \mathrm{TeV}$ together with example coverage by specific processes (jet and W, Z production). (right) A summary of effective DPI cross sections $\sigma_{\text {eff }}$ derived from a variety of processes studied at different hadron-collider experiments [21].

The broad agreement of the measurements with the predictions is thus a major triumph for the SM in general and QCD and the DGLAP formalism more specifically.

In the following, I will discuss a selection of recent measurements, starting with the most abundant processes involving only the strong interaction, moving on to processes involving the production of single electroweak bosons and a small selection of multi-boson processes before concluding with the current status of the studies of the Higgs boson.

\section{Soft QCD Processes}

The majority of $p p$ interactions take place at small energy scales, where $\alpha_{\mathrm{S}}$ is large and nonperturbative effects dominate. Effects of this "soft regime", such as inelastic minimum-bias interactions, often cannot be systematically computed through the usual perturbative QCD techniques. We thus need to resort to phenomenological models integrated in Monte Carlo (MC) tools like Pythia, Herwig or Epos [22, 23, 24] that are incorporate aspects of QCD and require tuning to data. Despite the fact that these $\mathrm{MC}$ tools are not directly connected to the SM Lagrangian, they are indispensable to the work of experimentalists. MC simulations are used to model the soft effects and their influence on measurements of processes at large energy scale, such as hadronisation of quarks and gluons and additional interactions beyond the hard scattering ("underlying event"). It remains a challenge to develop and constrain these models further such that non-perturbative effects will not limit the interpretation of LHC measurements in the future. Detailed measurements often show significant discrepancies to 
the model predictions. For example, the ALICE collaboration recently reported an enhanced production of strange hadrons in high-multiplicity $p p$ collisions [25], suggesting a lack of understanding of the mechanism in the current MC models.

Another interesting aspect is the existence of hard double-parton interactions (DPI), which despite taking place at perturbative energy scales point beyond our typical pQCD framework. In a simple model, the DPI interaction may factorize into two effectively independent partonparton scatters within the same $p p$ interaction, however the probability for the second interaction is adjusted by a factor $\sigma_{\text {inel }} / \sigma_{\text {eff }}$. Here $\sigma_{\text {inel }}$ refers to the total inelastic interaction cross section and $\sigma_{\text {eff }}$ is the so-called effective DPI cross section, a parameter to be determined in the experiment. In Fig. 2(right) an overview of measurements of $\sigma_{\text {eff }}$ from both TeVatron and LHC analyses is given. The current data set is still sparse and does not allow to determine a clear dependence on the centre-of-mass energy or the final states considered. Most analyses find a value for $\sigma_{\text {eff }}$ that is a significant factor $\sim 5$ below $\sigma_{\text {inel }}$. This possibly suggests a "hotspot" interpretation, where the existence of a first hard interaction increases the probability to find a second hard scatter. Interestingly the values of $\sigma_{\text {eff }}$ extracted from double quarkonia final states feature further reduced $\sigma_{\text {eff }}$ values, suggesting an enhanced correlation in these cases.

\section{$5 \quad$ Production of jets and heavy-flavour jets}

In the perturbative regime, the strong interaction of quarks and gluons leads to an abundant production of hadronic jets with high transverse momentum. The ATLAS and CMS collaborations have performed a series of measurements at energies of $\sqrt{s}=2.76-13 \mathrm{TeV}$ [26, 27, 28, 29, 30]. Figure 3(left) shows an overview of a typical measurement of inclusive jet cross sections double-differentially in the transverse momentum $p_{T}$ and rapidity $y$ of the jet together with a QCD prediction at NLO accuracy, supplemented by corrections for electroweak and non-perturbative effects, where the latter include corrections of underlying event and hadronisation. Inclusive jet measurements have the potential to cover almost the full kinematic plane of accessible parton dynamics, as can be appreciated in Fig. 2(left). The data typically agrees with the QCD predictions to $5-10 \%$ as shown in Fig. 3(right), which is truly remarkable given the wide kinematic range and many orders of magnitude in the cross section probed. The cross section at low $p_{T}$ is gigantic in comparison to the luminosity the LHC is able to deliver and measurements are typically performed using small data sets taken at low instantaneous luminosity.

The measurements at higher $p_{T}>100 \mathrm{GeV}$, where the effects of non-perturbative corrections are progressively less important, may provide information on the gluon PDF at larger $x$ and are routinely used in PDF fits. The recent completion of calculation of the NNLO corrections [31, 32] is a milestone to put the interpretation of the data on a more solid footing and promises a reduction of the theoretical uncertainties to a level less than the PDF and the measurement uncertainties. However, contrary to the common expectation, a significant uncertainty from scale variations remains at NNLO. In addition, the choice of the scale as either the leading jet $p_{T}$ or the individual jet $p_{T}$ has a significant impact on the visual agreement with the data, where the latter choice even leads to a worse agreement with the data than the NLO prediction. Both of these features can be appreciated in Fig. 4 in comparison to a recent measurement. Future studies of the theory calculation and definition of the experimental measurement may be able to resolve this issue in the interpretation of the data. 

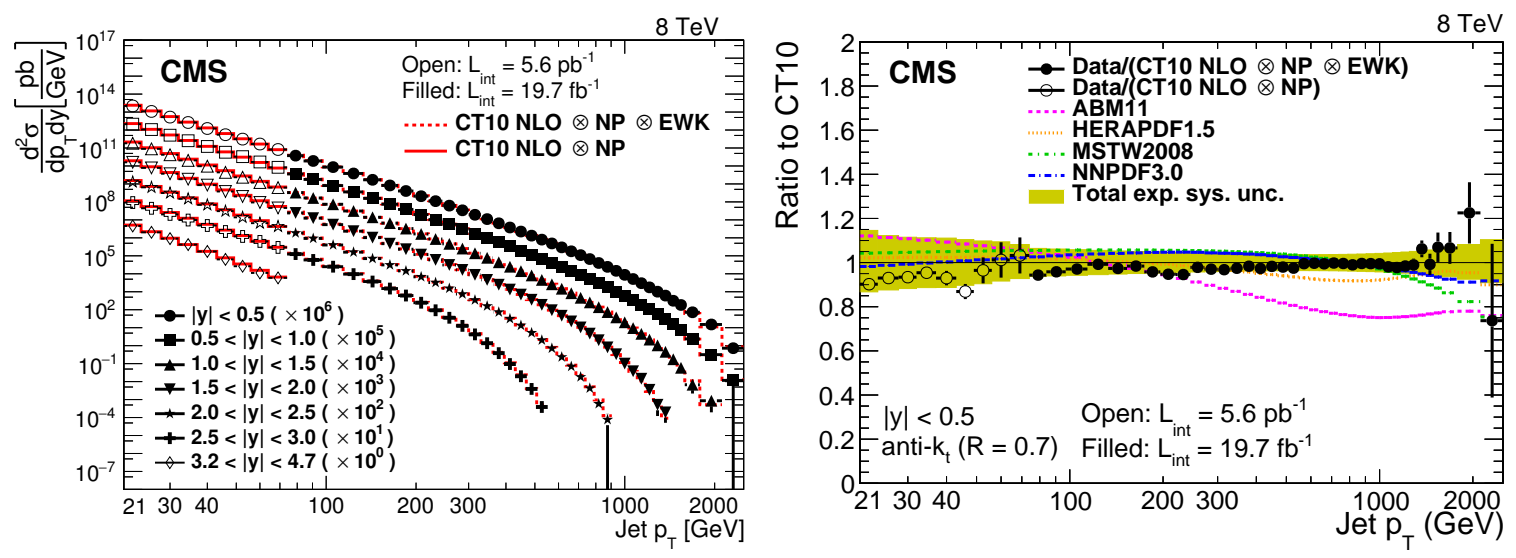

Figure 3: Double-differential inclusive jet cross-section measurement at $\sqrt{s}=8 \mathrm{TeV}$ from Ref. [26]. (left) gives an overview of all data points compared to the NLO QCD prediction. (right) shows the ratio of the data at central rapidity to NLO predictions with different PDF sets.

An interesting extension to inclusive hadronic jets are measurements of jets produced in the fragmentation of heavy quarks. The production of charm and beauty hadrons has been performed down to very low transverse momentum and in the forward region by the LHCb collaboration [33]. This data is sensitive to the gluon density at very low $x \sim 10^{-6}$, a region not probed by any other collider data so far. However, the theoretical uncertainties on the NLO predictions are large, up to $\sim 30 \%$ and the interpretation relies on the cancellation of these large uncertainties in suitably defined ratios [34, 35.

The LHC has moved the study of top-quark pair production through the strong interaction to a new level compared to the TeVatron, where the top-quark was discovered and many studies were pioneered. The remainder of this chapter will not be able to do justice to this active field, but just give a glimpse of the more recent results. Predictions for top-quark pair production are available up to NNLO QCD differentially in the stable top-quark kinematics [36] and these are generally seen to describe the data well, as shown in Figure 5(left) for a recent measurement differential in the $t \bar{t}$-system mass. The measurement of the top-quark mass has reached a precision of about $0.5 \mathrm{GeV}[37,38$. At this level it is likely that measurements of the "MC mass" differ from the (theoretically desired) "pole mass" for example due to nonperturbative and colour-reconnection effects. Methods exploiting the total [39] or differential cross-section measurements [40] are better controlled with respect to non-perturbative effects and have recently reached uncertainties of well below $2 \mathrm{GeV}$ as shown in Figure 5(right), typically limited by the uncertainties on the PDFs and missing higher-order corrections.

Finally, the production of jets may be used to probe the strong coupling $\alpha_{\mathrm{S}}$, which is obviously "a crucial ingredient of perturbative QCD" 42 and in fact the least-well known of the fundamental couplings with a precision of about $1 \%$ at a scale of the $Z$-boson mass. Unfortunately it is not possible to extract $\alpha_{\mathrm{S}}\left(m_{Z}\right)$ from LHC jet measurements with competitive precision. Instead, the data may be used to probe $\alpha_{\mathrm{S}}$ at very large scales of up to $2 \mathrm{TeV}$ [27. Determinations from the $t \bar{t}$ cross section have been shown to reach an improved precision compared to inclusive jets [43, 44]. The uncertainty in $\alpha_{\mathrm{S}}$ is important for several LHC predictions, such as Higgs-boson production. The size of the uncertainty in $\alpha_{\mathrm{S}}$ is a topic of debate and Guido Altarelli certainly had an opinion on this issue, advocating caution to 

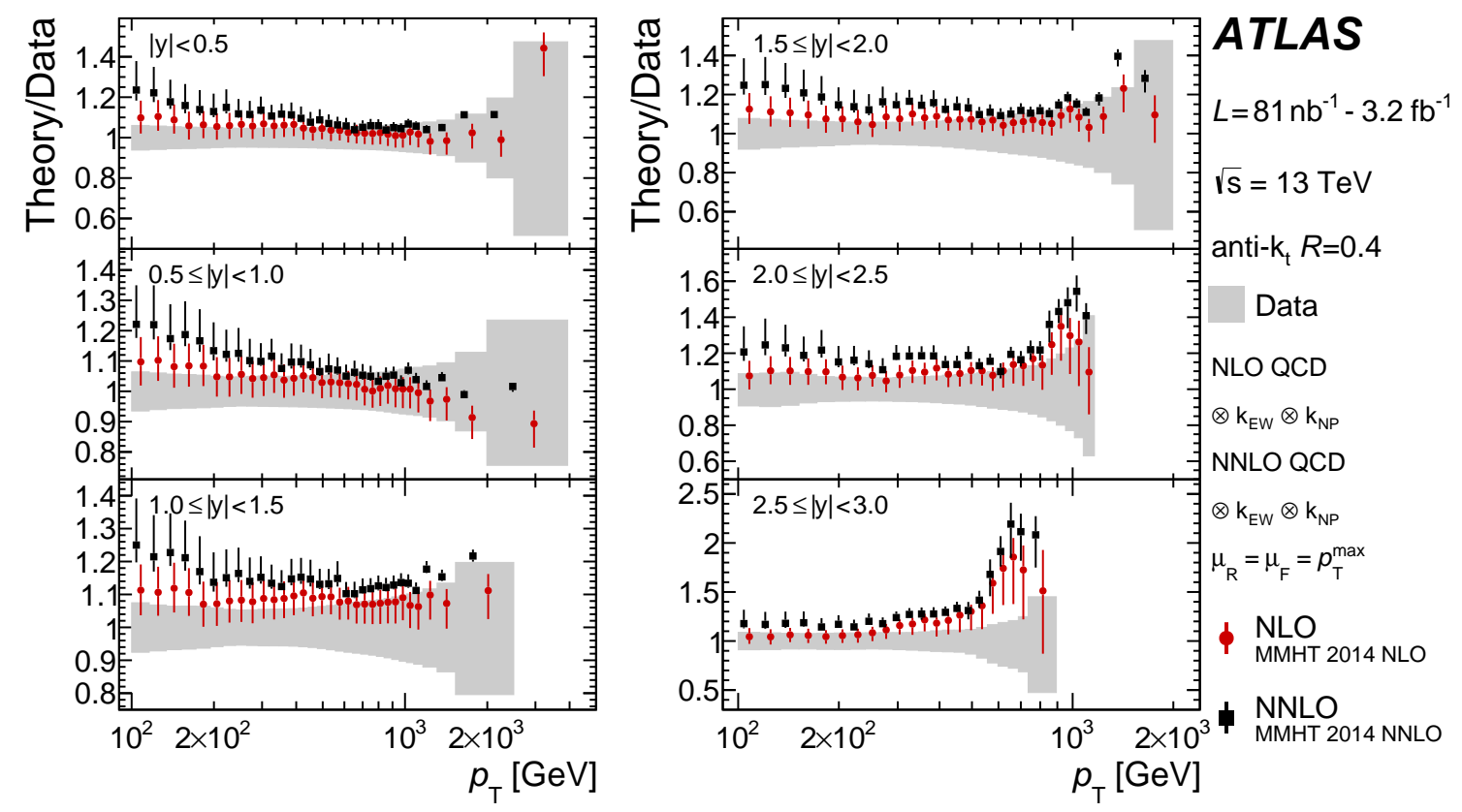

Figure 4: Double-differential inclusive jet cross-section measurement at $\sqrt{s}=13 \mathrm{TeV}$ from Ref. [29] compared to NLO and NNLO QCD predictions with the scale set by the leading jet $p_{T}$.

the estimates of theory uncertainties [42].

\section{Di-lepton production in the Drell-Yan Process}

Di-lepton production in hadron collisions through the Drell-Yan (DY) process [16] has played a fundamental role in establishing the SM. It is here considered to include both the neutral current $q \bar{q} \rightarrow Z / \gamma^{*} \rightarrow \ell^{+} \ell^{-}$as well as the charged current $q \bar{q}^{\prime} \rightarrow W^{ \pm} \rightarrow \ell^{ \pm} \stackrel{(-)}{\nu}$ reactions. Of critical importance was the discovery of the heavy intermediate vector bosons $W$ and $Z$ by the UA1 and UA2 experiments [45, 46, 47, 48] in DY reactions. Beyond these discoveries, the DY process was a test-bed to develop the procedure to systematically calculate and improve cross sections in hadron-collisions through the factorization theorem and an expansion in orders of $\alpha_{\mathrm{S}}$. In fact, this process remains one of the few examples in hadron-hadron collisions, where factorization has been rigorously proven to hold [49, 50].

Each major step forward in the theory calculation typically took a decade. The first step beyond the initial leading order calculation came through major contributions of the "Rome group" with Guido Altarelli [51, who completed "one of the first calculations of NLO corrections in QCD" in 1978/79 [52, 15, 53, 54]. A critical part was to treat the PDFs and their evolution in a consistent way in DIS and DY reactions. The NLO corrections "turned out to be surprisingly large" [51, necessitating calculations beyond NLO. The NNLO corrections were completed in the early 1990s [55, 56, 57] and the fully differential NNLO predictions became available until about 2010 [58, 59, 60, 61].

Figure 6 shows the number of citations of the original paper by Drell and Yan as a function of time. This reflects the initial interest in the process to establish the SM in the 1980s, as well 

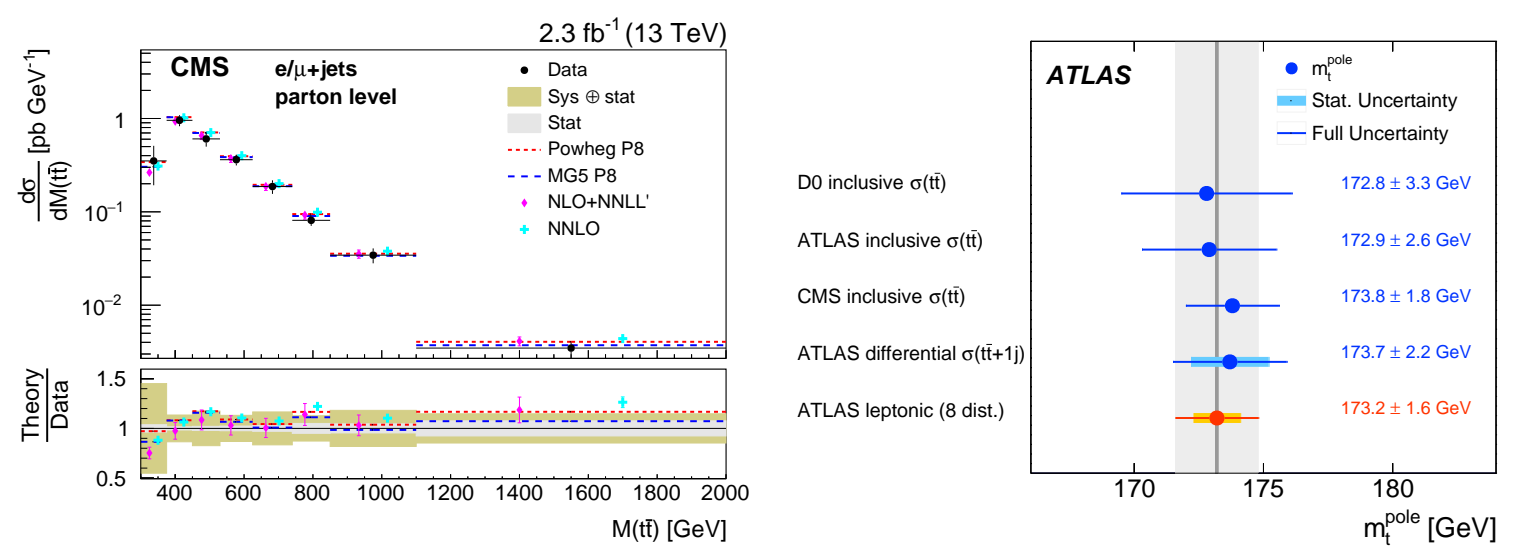

Figure 5: Example of recent measurements with top-quarks: (left) shows a measurement of the differential cross section for $t \bar{t}$ production at $\sqrt{s}=13 \mathrm{TeV}$ compared to a selection of predictions by MC programs and fixed-order calculations [41. (right) shows a recent compilation of top-quark mass extractions using cross-section data 40]

as the more recent "resurrection" that is closely linked to new analyses of this process at the LHC. This renewed interest has diverse reasons, which includes the study of PDFs, precision measurements of SM parameters as the $W$-boson mass and the effective weak mixing angle $\sin ^{2} \theta_{W}$, and the background to searches beyond the SM as for example particle candidates for dark matter [62]. The measurements are facilitated by large samples of tens of millions of events reconstructed with small backgrounds and well-understood detector performance for the final-state leptons.

Measurements of differential cross sections and distributions for $Z / \gamma^{*}$ and $W^{ \pm}$production have been performed by the ATLAS, CMS and LHCb collaboration in $p p$ collisions at $\sqrt{s}=2.76-13 \mathrm{TeV}$ and as a function of many kinematic variables. The classical variables to constrain PDFs are the di-lepton rapidity and mass, $y_{\ell \ell}$ and $m_{\ell \ell}$ for $Z / \gamma^{*}$ and the lepton rapidity $\eta_{\ell}$ for $W^{ \pm}$production with some recent examples given by Refs. 63, 64, 65, 66, 67, 68. Figure 7 shows two examples of such measurements compared to state-of-the-art cross-section calculations with different PDF sets. The measurements have reached an accuracy of a percent or better, which is below the uncertainties of a typical PDF set and often also below the uncertainty of the NNLO theory calculation. The fact that these precise $W^{ \pm}$and $Z / \gamma^{*}$ cross-section data generally agree with the predictions, is another testament to the success of our understanding of perturbative QCD.

Such data can now be included in PDF analyses based on either the HERA DIS data set [69] or global PDF fits [70, 71, 72, 73] to improve our knowledge on the proton structure in broadly speaking three areas. Firstly, the LHCb data gives unique information on PDFs at very low Bjorken $x \sim 10^{-4}$ or very large $x \sim 0.5$, a range where the information of the HERA data set is limited and information at high $x$ from fixed-target data may not be fully reliable. Second, as illustrated in Figure 8(left), data on $W^{ \pm}$-boson production helps to improve the information on low and intermediate $x$ valence quarks distributions. Finally, $Z$ production at the LHC at central rapidity has a significant contribution from strange quarks, which are only constrained from charm production in neutrino-nucleon DIS on heavy targets at somewhat larger $x$. As demonstrated in Figure 8(right), the LHC data has here a unique impact and strongly hints at a larger strange-quark density than previously inferred from the neutrino 


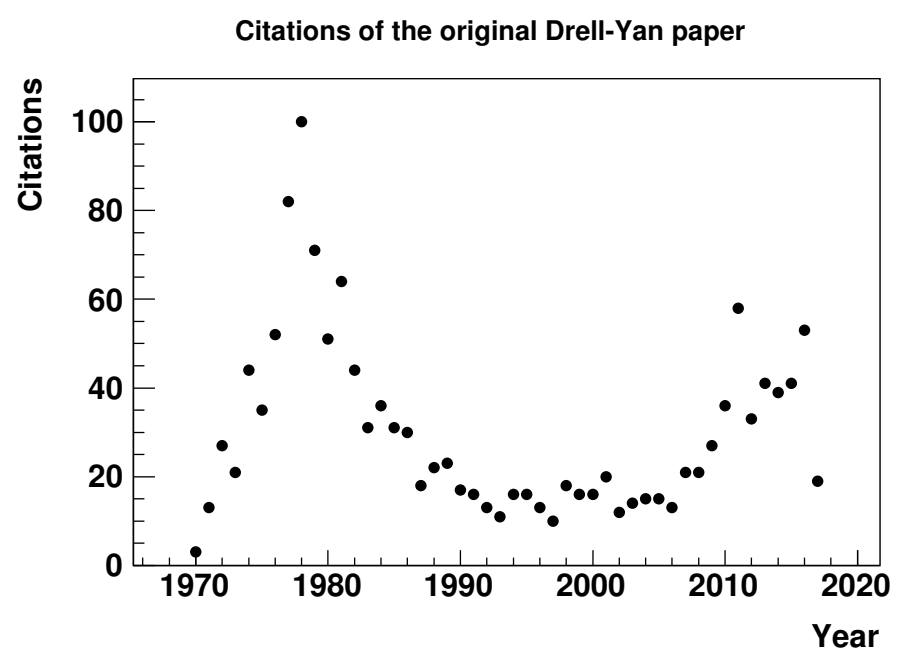

Figure 6: History of citations of the original paper on the Drell-Yan process [16] taken from inspire.net.

data.

Another key variable is the transverse momentum of the boson, which poses an interesting theoretical challenge and has implications for many studies at the LHC. The distribution is more easily measured in the neutral current process as the di-lepton $p_{T, \ell \ell}$, see e.g. Refs. 77 , 78, 79]. The spectrum can be roughly separated into two regimes at a transverse momentum of above and below half the boson mass. The higher- $p_{T, \ell \ell}$ region may be described by a fixed-order calculation, while an all-order resummation calculation is required to describe the lower- $p_{T, \ell \ell}$ domain that is dominated by multiple soft-gluon emission and possibly also influenced by non-perturbative effects. Figure 9 shows the high $p_{T, \ell \ell}>20 \mathrm{GeV}$ part of a measurement differential in both the di-lepton transverse momentum and mass compared to a very recent NNLO fixed-order calculation for the $p p \rightarrow Z+$ jet process at order $\alpha_{S}^{3}$ [80, 81. The correction to the $\alpha_{S}^{2}$ calculation is seen to be significant, although within the uncertainty estimate in the range $p_{T, \ell \ell} \gtrsim m_{\ell \ell}$. In parts of the phase space, especially near the $Z$-boson resonance, the data is more precise even than the $\alpha_{S}^{3}$ prediction. There remains a tension between the data and the prediction that may be related to the PDFs and can possibly be improved by including the data in a PDF analysis as discussed in Ref. [82.

The large data sets of $Z / \gamma^{*}$ and $W^{ \pm}$can be used for precision measurements of the effective weak mixing angle $\sin ^{2} \theta_{W}$ and the $W$-boson mass, respectively. These measurements are interesting to pin down the fit of precision electroweak observables [84, 85] and search indirectly for deviations from the SM [86]. The $\sin ^{2} \theta_{W}$ measurement exploits the change of the "Forward-Backward Asymmetry" in the $q \bar{q} \rightarrow Z / \gamma^{*} \rightarrow \ell^{-} \ell^{+}$process in the vicinity of the $Z$ resonance. The $W$-boson mass can be extracted through minuscule changes in the shapes of kinematic variables such as the lepton $p_{T}$ in $W$ events.

These electroweak measurements require an extreme experimental precision, for example the lepton momentum calibration has to be understood to the $10^{-4}$ level. Even more critical is the control of uncertainties related to the modelling of the sensitive distributions with respect to QCD effects related to PDFs, heavy-flavour initiated processes and the transversemomentum spectrum of the bosons. The LHC environment poses such a challenge, that 

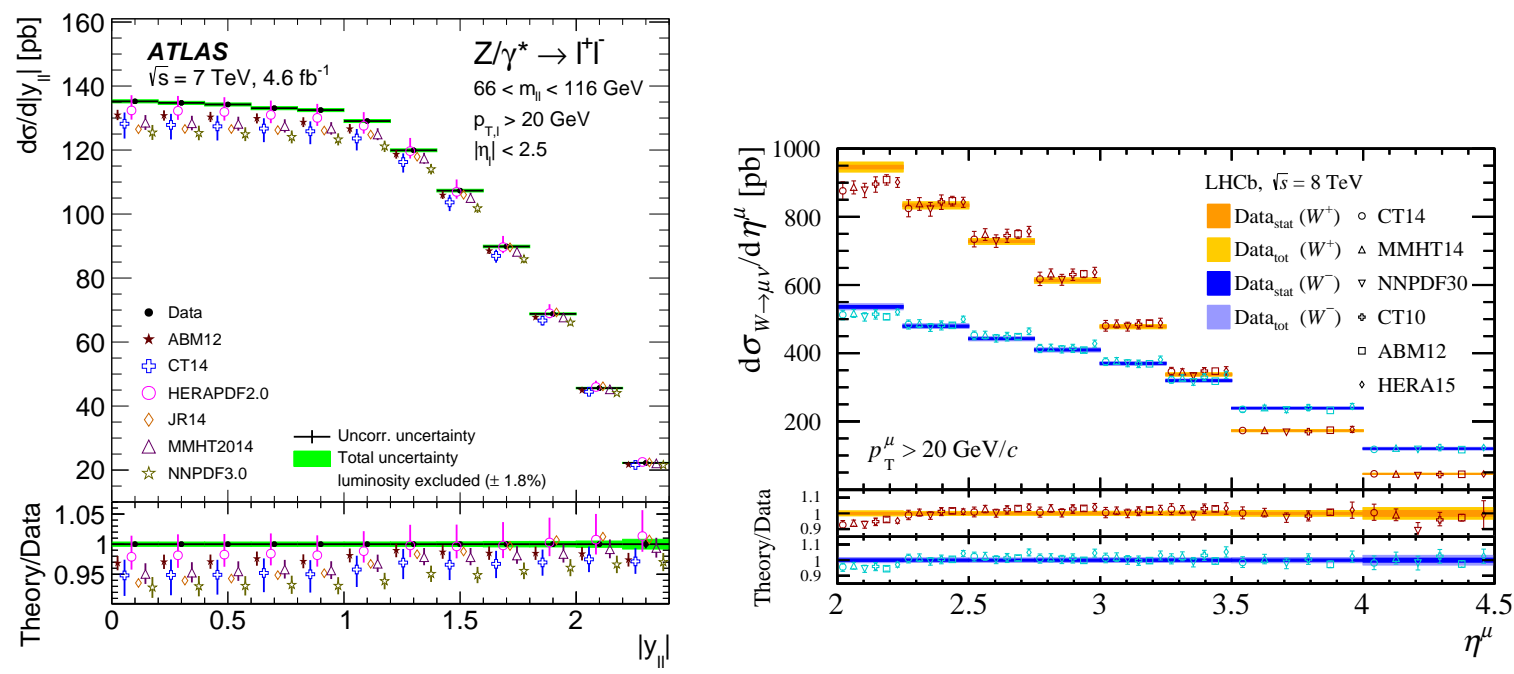

Figure 7: Two examples of recent Drell-Yan measurements at the LHC compared to NNLO QCD predictions with a variety of different PDF sets. (left) shows a measurement of the differential $Z / \gamma^{*}$ cross section as a function of the di-lepton rapidity y y at $\sqrt{s}=7 \mathrm{TeV}$ 63] (right) shows a measurement of $W^{+}$and $W^{-}$production as a function of the lepton pseudo-rapidity $\eta_{\ell}$ [67]

Guido Altarelli as many of his peers was skeptical about the feasibility of such an enterprise, stating that "... anyone [who] wants to measure the $W$ mass at the LHC with high precision, better he commits suicide ..." [87].

The achievements of the first competitive measurements of the $W$-boson mass [88] and $\sin ^{2} \theta_{W}[89$ in spite of the major effort are therefore all the more remarkable. Figure 10 (left) gives an overview of $\sin ^{2} \theta_{W}$ from $e^{+} e^{-}$colliders, the TeVatron and the LHC. The most recent CMS measurement with the $\sqrt{s}=8 \mathrm{TeV}$ data set reaches a precision similar to the TeVatron experiments and "just" a factor two worse than the LEP and SLD results. In Figure 10 (right) an overview of measurements of the $W$-boson mass is shown including the recent ATLAS determination using the $\sqrt{s}=7 \mathrm{TeV}$ data set, which has reached the same uncertainty as the previously best measurement by CDF.

\section{Multiboson production and electroweak processes}

In addition to the study of the simpler and abundant processes, the LHC allows also the measurements of rarer and more complex final states. In this section we will discuss processes that go beyond the leading order in the electroweak couplings, where either two or three bosons $V=W, Z, \gamma$ are produced or two of these bosons "fuse" (vector boson fusion, VBF) or "scatter" (vector boson scattering, VBS). The diagrams involved contain vertices sensitive to the triple and quartic couplings of the electroweak bosons, which are non-trivial in the SM due to the non-Abelian $U(1)_{Y} \times S U(2)_{L}$ structure, which is "unbroken in the interaction vertices, ... [but] is instead badly broken in the masses" [1] by the Higgs mechanism. In the SM the interplay of various diagrams, such as those containing $\gamma W W$ and $Z W W$ vertices, guarantees a result that does not violate unitarity. Another well-known example is the scattering of 

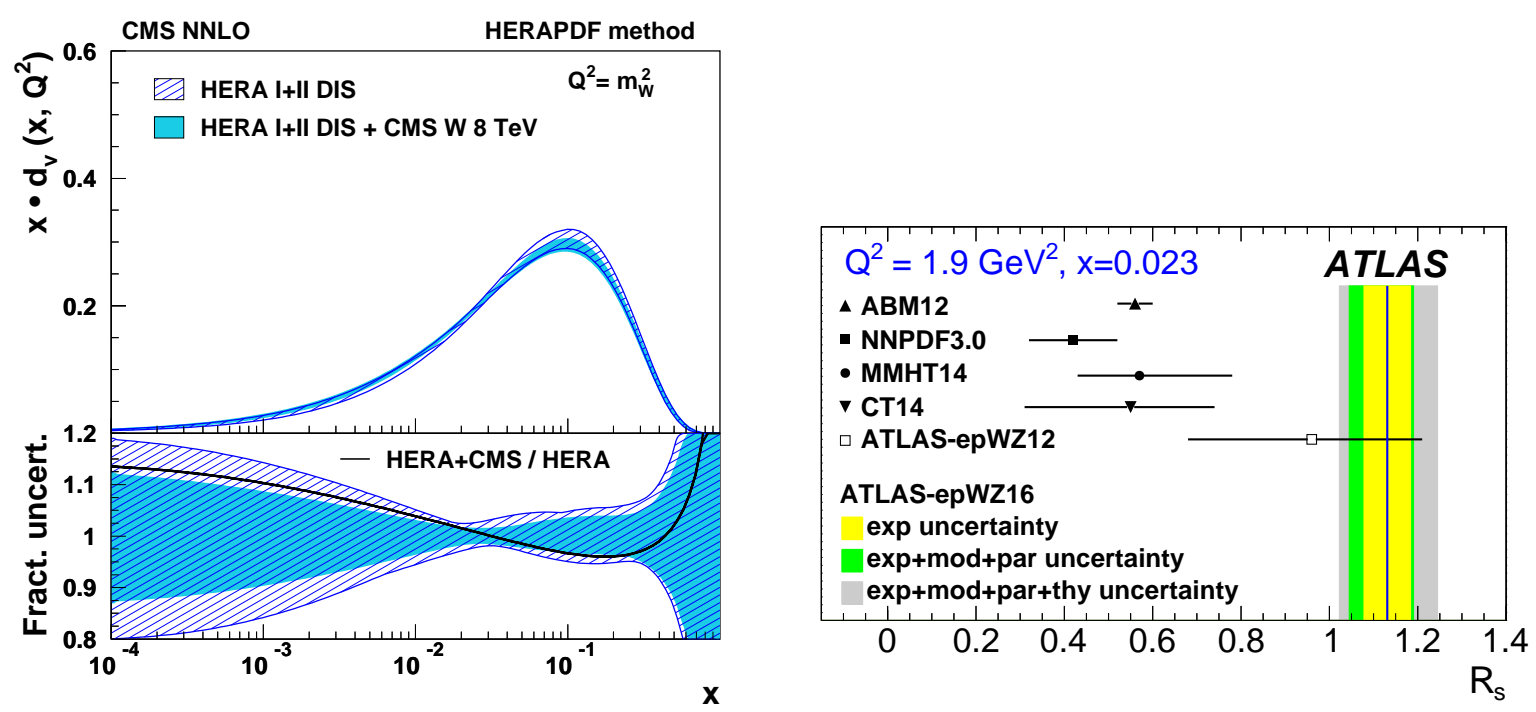

Figure 8: Two examples showing the impact of LHC $W^{ \pm}$and $Z / \gamma^{*}$ production data on the knowledge on the proton PDFs. (left) shows a study of including differential $W^{ \pm}$data in a PDF fit based on HERA DIS data, comparing the uncertainties on the $d$-valence density with and without the $W^{ \pm}$data [66] (right) show the ratio $R_{s}=(s+\bar{s}) /(\bar{u}+\bar{d})$ as a measure of the size strange-quark distribution compared to the up- and down-quark sea. The result from a fit with LHC $Z / \gamma^{*}$ and $W^{ \pm}$data labelled as "ATLAS-epWZ16" 63] is compared to the prediction of other PDF fits [74, 75, 70, 71, 76].

longitudinally polarized massive vector bosons, e.g. $W_{L} W_{L} \rightarrow W_{L} W_{L}$, where unitarity is violated already at about $1 \mathrm{TeV}$ without the contribution from the Higgs boson [90, 91, 92. Any deviation from the SM predictions would imply physics with new forces or particles. 2

In Figure 11(left) an overview of measurements of di-boson cross sections is given. All possible combinations of $V V$ processes have been measured, with the majority of measurements considering the decays with (charged) leptons of the massive $W$ or $Z$ bosons. The precision has reached a remarkable level of about $5 \%$ for several of the combinations. An interesting feature, visible even on this coarse overview, are the large differences between the theory calculations at NLO and NNLO QCD, where the NNLO predictions are in much better agreement with the data. Such features were noticed already in the early analyses of the $W \gamma$ [93, and later explained by large NNLO corrections 94. A similar feature is also noticeable in the $W Z$ final state [95, which shares with the $W \gamma$ process the feature of a "radiation-zero" suppressed LO prediction with large higher-order QCD corrections.

For neutral combinations such as $\gamma \gamma, W W$ and $Z Z$, the higher-order QCD corrections have also been found to be large, mainly because of the gluon-initiated contribution $g g \rightarrow V V$ through a box diagram, which appear first as part of the NNLO $\left(\alpha_{S}^{2}\right)$ calculation and receive large $\alpha_{S}^{3}$ corrections [96]. The final state with four charged leptons, $4 \ell(\ell=e, \mu)$, is particularly instructive, as the excellent lepton identification capabilities of the LHC detectors allow a

\footnotetext{
${ }^{2}$ Similarly, it is interesting to study the electroweak properties of the top-quark, which may have a special role in the process of electroweak symmetry breaking due to its large mass. Such studies can be performed e.g. through electroweak production of single top-quarks or the production of top-quarks in association with electroweak bosons $t \bar{t} V$ or $t V$, but a detailed discussion is beyond the scope of this section.
} 


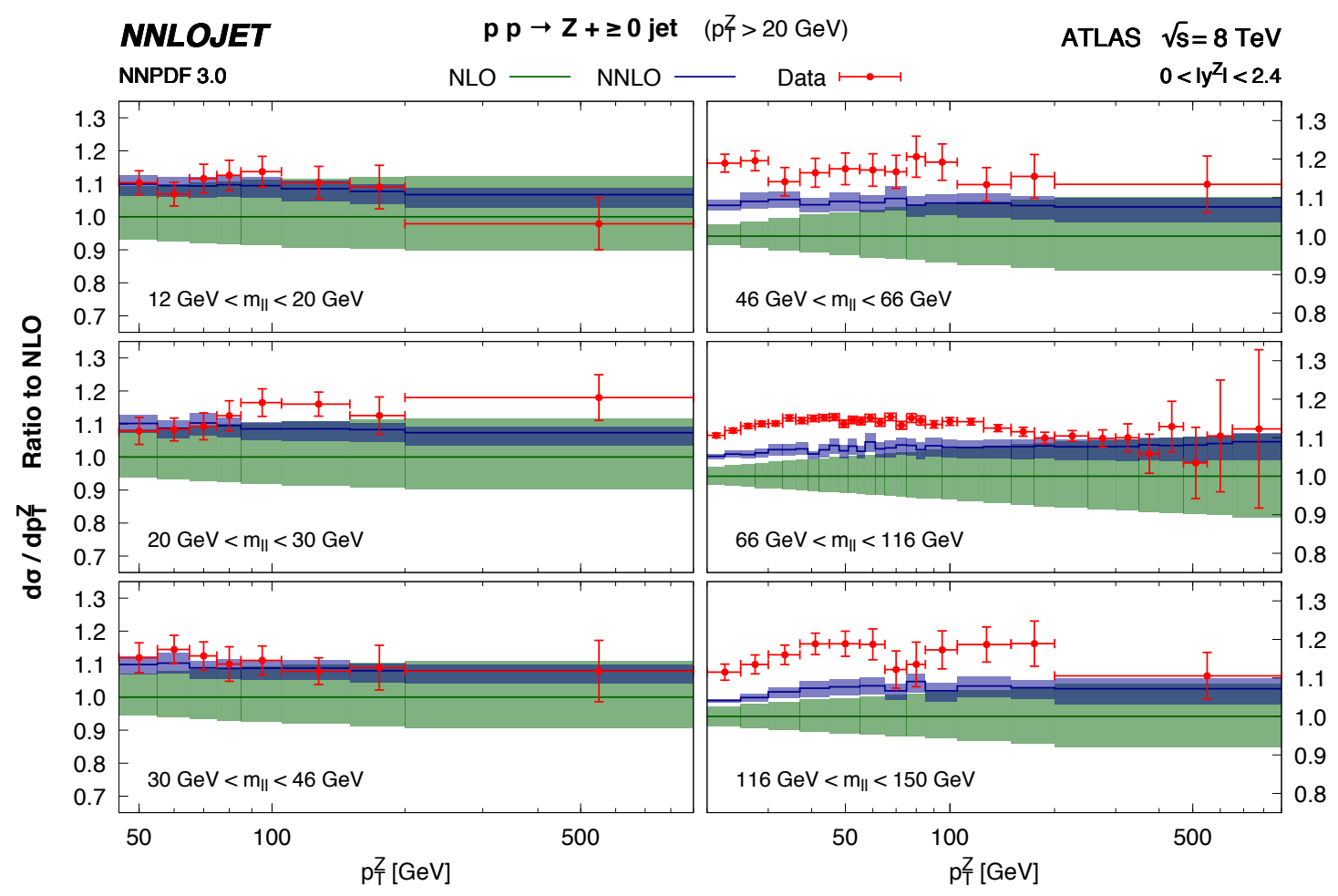

Figure 9: Double-differential di-lepton cross-section measurement as a function of mass and transverse momentum at $\sqrt{s}=8 \mathrm{TeV}$ compared to a fixed-order NNLO calculation [83].

full reconstruction without significant backgrounds in an wide range of four-lepton invariant masses from about $m_{4 \ell}=80-1000 \mathrm{GeV}$ [97, 98, although the rate is low because of the small branching of the $Z$ boson to two charged leptons. As illustrated in Figure 11(right), this distribution contains a host of interesting features: a resonance at the mass of the $Z$ boson from the radiative decay $Z \rightarrow 4 \ell$, a resonance at the mass of the Higgs boson from its golden decay $H \rightarrow Z Z^{*} \rightarrow 4 \ell$, a threshold at twice the $Z$ boson mass for the production of two onshell $Z$ bosons, and finally a negative interference of gluon-induced $Z Z$-production diagrams with and without the Higgs boson, important especially above $m_{4 \ell}>2 m_{\text {top }}$, which is also sensitive to the width of the Higgs boson [99.

In contrast to the measurements of di-boson final states, which is entering the precision phase, the searches for tri-boson processes will require more luminosity, with the exception of the $W \gamma \gamma$ and $Z \gamma \gamma$ final states, for which evidence and observations have been reported, respectively [100, 101, 102].

The electroweak production of one or two electroweak bosons in the VBF and VBS processes, respectively, tests the self-interactions of three or four electroweak bosons. Experimentally these are challenging to measure due to small cross sections and large backgrounds from processes mediated by the strong interaction, which are typically separated from the signal through "tagging" the signature by a rapidity gap and a high-mass dijet system [103. as illustrated for the case of electroweak $Z j j$ production in Figure 12(left). An overview of the status of the field is shown in Figure 12(right). At this point in time, the electroweak production of $W$ and $Z$ bosons has been observed with high significance and studied in de- 

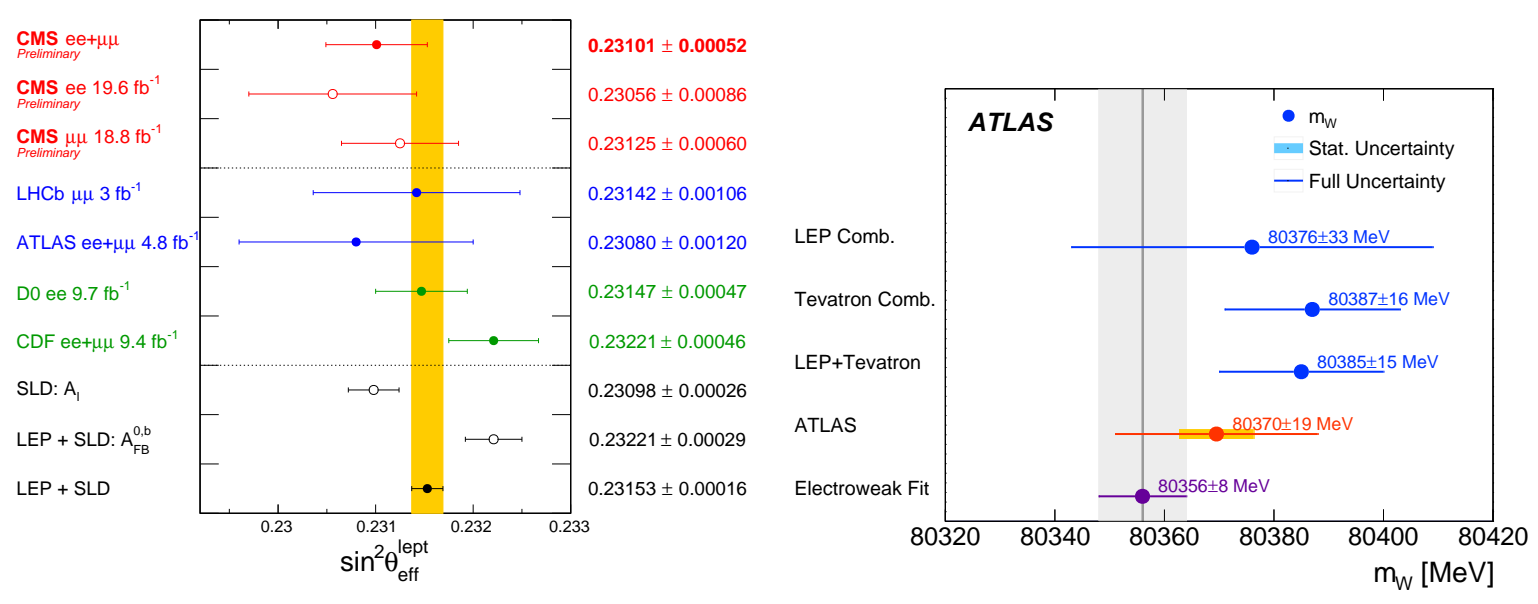

Figure 10: Overview of measurements of precision electroweak parameters at different colliders for (left) the effective weak mixing angle $\sin ^{2} \theta_{W}$ [89] and (right) the mass of the $W$ boson [88.

tail [104, 105, 106, 107, 108, For VBS processes, most analyses have not yet reached the observation stage. A significant exception is the VBS production of same-sign $W^{ \pm} W^{ \pm} j j$, the golden VBS process due to low strong-interaction backgrounds, where recently an observation could be reported [109].

\section{Status of the studies of the Higgs boson}

The Higgs boson was the last part of the Standard Model to be discovered. Until then, the existence of the Higgs sector was seen as "a mere conjecture" by many, including Guido Altarelli [1]. Thanks to the well-working LHC and its experiments, the discovery of "a" Higgs boson with a mass near $125 \mathrm{GeV}$ could be announced already in July 2012, delivering on the promise that "the LHC [was] designed to solve the Higgs problem" [1]. Since then, the properties have been studied further using the data collected in Run 1 and now anew using the Run 2 data. Already the Run 1 data allowed a precise measurement of the mass to $m_{H}=$ $125.09 \pm 0.24 \mathrm{GeV}$ [110], fixing the last free parameter of the SM. Also the rates for the main production and decay rates could be confirmed to be as predicted by the SM within a typical experimental precision of $20-50 \%$. The overall agreement of Higgs production and decay with the SM expectation may be summarised with the signal strength of $\mu=1.09 \pm 0.11$ [111], well compatible with the SM expectation of 1 . The quantum numbers were confirmed to be $J^{C P}=0^{++}[112$, 113] with high probability. As shown Figure 13(left), the Run 1 data established a reasonable agreement with the expectation, that the masses of fermions and bosons are indeed given by Yukawa couplings proportional to the particle masses, although also here the experimental precision was typically just a few $10 \%$.

As commented by Guido Altarelli, the good agreement of the measurements with the minimal version of the SM and the Englert-Brout-Higgs mechanism and at the same time an absence of other evidence for physics beyond the Standard Model, poses new theoretical challenges, as the Higgs boson is "simple yet so unnatural" [5]. One of the paths to elucidate the mechanism of electroweak symmetry breaking further are precision measurements of the properties of the Higgs boson. With the availability of the Run 2 data, Higgs measurements 

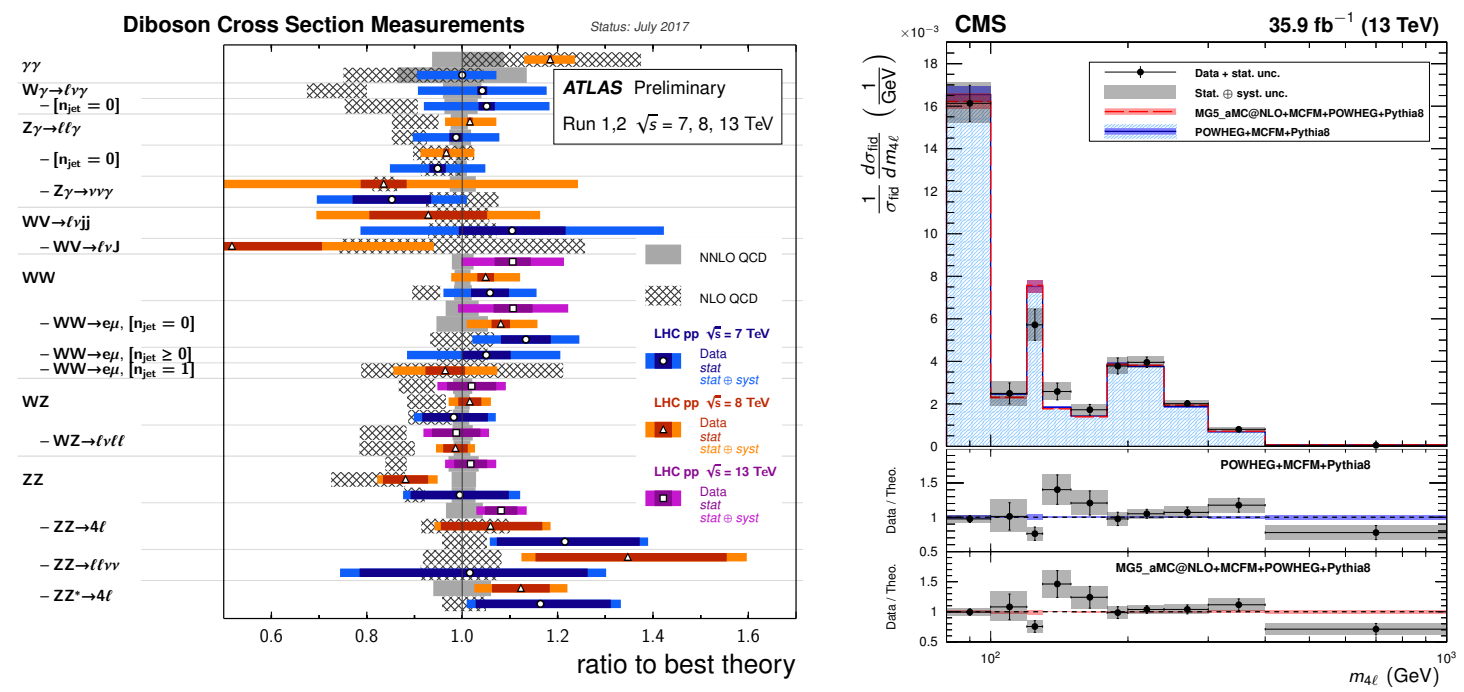

Figure 11: (left) A summary of diboson cross-section measurements by the ATLAS collaboration [19]. Measurements are compared to SM theory predictions calculated at NLO (hatched) and NNLO QCD (solid, where available). (right) The four-lepton invariant mass spectrum, $m_{4 \ell}$, compared to the SM prediction including the processes $Z \rightarrow 4 \ell, H \rightarrow 4 \ell$ and $Z Z^{(*)} \rightarrow 4 \ell[98$.
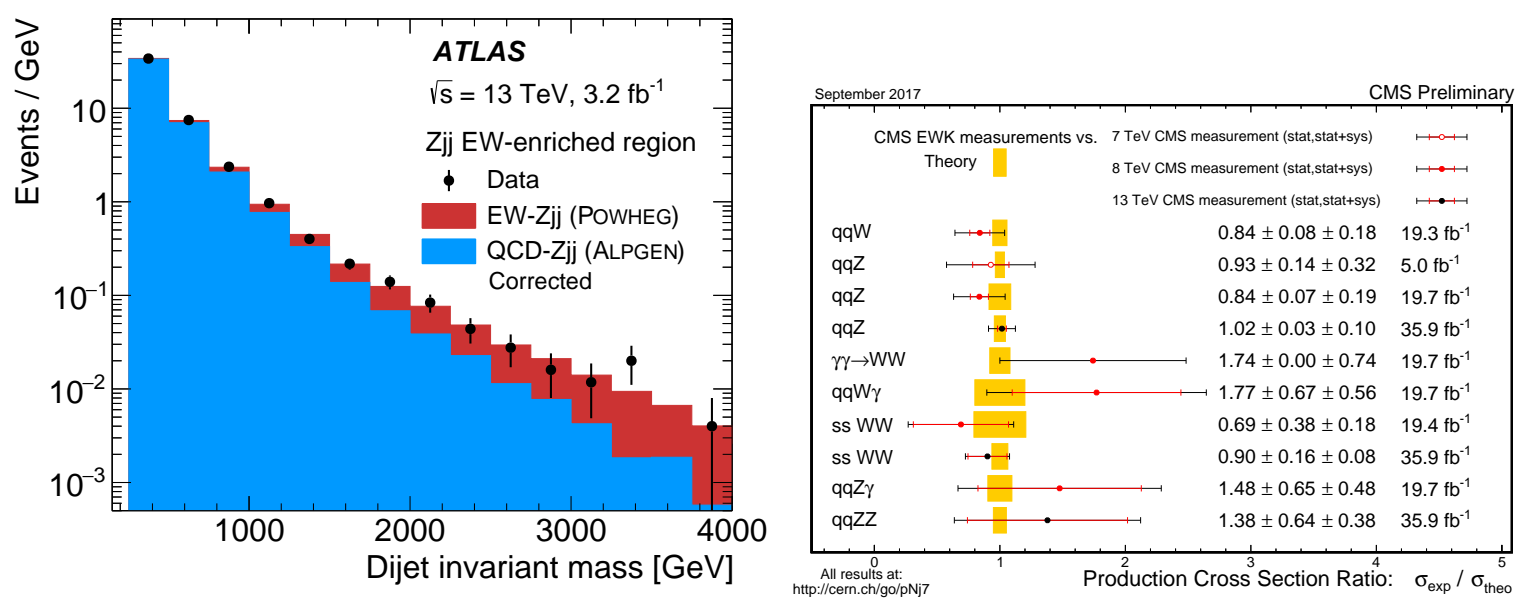

Figure 12: (left) The dijet invariant mass spectrum in $Z j j$ events, where little hadronic activity is required in the rapidity region between the two tag-jets. The electroweak VBF process is clearly separated from the strong-interaction background at large $m_{j j}$. [108, (right) A summary for the electroweak production of single or dibosons by the CMS collaboration [20]. 

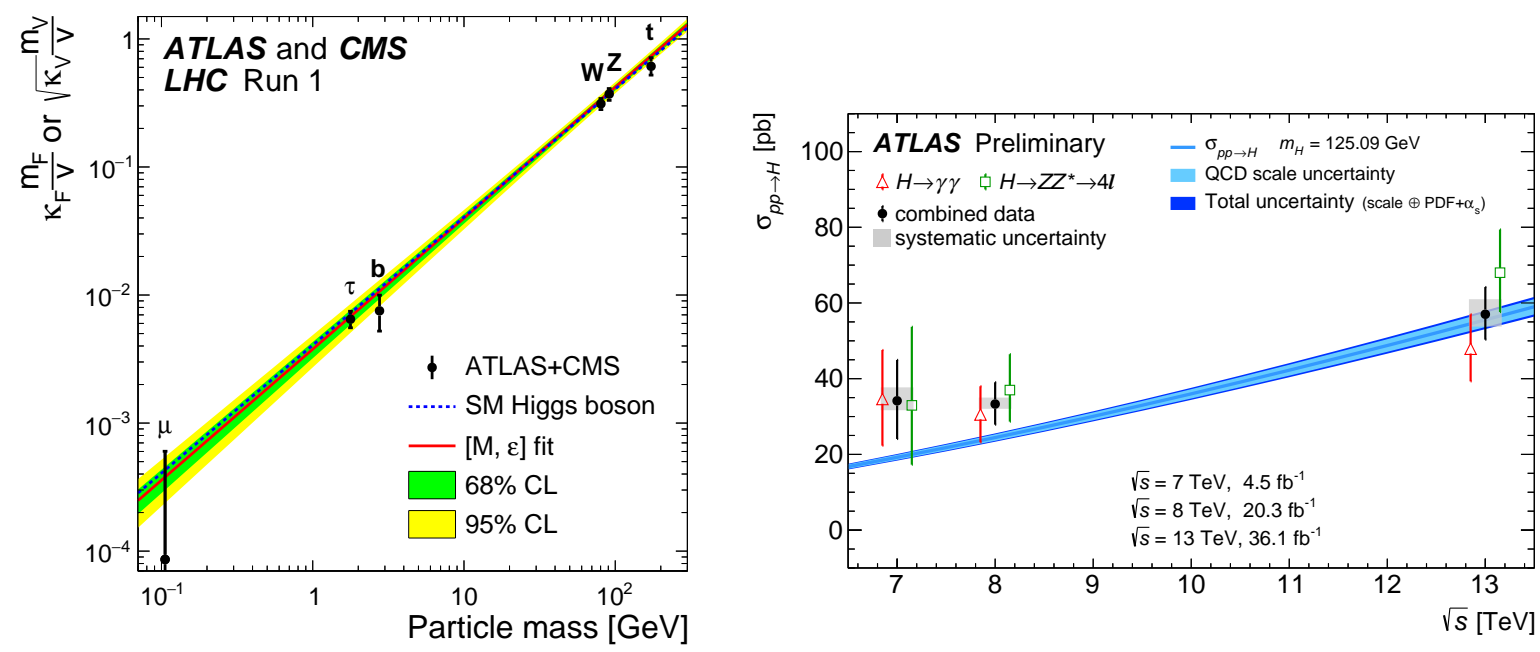

Figure 13: (left) Measurements of the coupling of the Higgs-boson to fermions and vector bosons as a function of the particle mass compared to the SM expectation. [111] (right) Combination of Higgs-boson production cross sections measured in the $H \rightarrow 4 \ell$ and $H \rightarrow \gamma \gamma$ decay channels at centre-of-mass energies of $\sqrt{s}=7,8,13 \mathrm{TeV}$ compared to the SM prediction [114].

have entered now a new phase. Exploiting the rarer but well-reconstructed decays $H \rightarrow$ $Z Z^{*} \rightarrow 4 \ell$ and $H \rightarrow \gamma \gamma$ of just one experiment, the total production cross section is already now measured at a precision of about $10 \%$ as shown in Figure 13(right), which is similar to the full Run 1 combination of all ATLAS and CMS data. Very recently, ATLAS and CMS have been able to report evidence for the decay $H \rightarrow b \bar{b}[115,116]$ and for Higgs-boson production in association with top-quark pairs $t \bar{t} H$ [117, 118. At this stage the results are compatible with the SM predictions with a precision of $30-40 \%$. These analyses are very complex and make use of the full abilities of the detectors, as well as mondern simulation and analysis techniques to control the large backgrounds.

In addition to more data, the precise measurements of Higgs-boson properties depend critically on a further improved understanding of the QCD aspects, both for the signal and background processes. A recent highlight was the completion of the $\mathrm{N}^{3} \mathrm{LO}$ calculation for the dominant gluon-fusion process [119]. While this significantly reduces the theoretical uncertainty in this particular calculation, uncertainties from the gluon PDF, the strong coupling $\alpha_{\mathrm{S}}$, as well as the missing $\mathrm{N}^{3} \mathrm{LO}$ corrections to the PDF evolution and DIS, DY and other relevant cross sections will be a challenge for the next years.

\section{Conclusion}

Since the year 2010 the LHC and the large experimental collaborations have successfully delivered a detailed picture of the fundamental constituents of matter and interactions at the electroweak scale and beyond into the TeV range. As promised, the LHC solved the "Higgs problem": the last missing piece of the Standard Model was discovered and the work is ongoing to measure the properties of the newly discovered particle. The Standard Model as we know it today is often discussed as something that is "unnatural", just a "low energy 
effective theory" and that "just describes $5 \%$ of the energy content of the universe", yet it is "excessively" successful. Guido Altarelli was hopeful that there could be a fundamental breakthrough out of this "puzzling situation". Meanwhile we should also celebrate the achievements of the SM which was built in decades of systematic work of theorists and experimentalists and which is able to describe the diverse LHC data.

The work ahead for the LHC community is certainly a challenge, but also full of exciting possibilities. The abundant and rich data provided by the LHC collisions can and will be analyzed in diverse ways to deepen our understanding of the strong and electroweak interactions. In many measurements and searches we enter the "precision regime" as we accumulate large data sets and continue to improve the understanding of our detectors. The methodology to understand the proton structure and calculate predictions in QCD have advanced to reach the percent level, which is truly remarkable given the inherent complexity.

Guido Altarelli was often skeptical what concerned the theoretical accuracy of QCD predictions which we experimentalists rely on so critically and which we love to challenge. It would be interesting to learn Guido's reaction to achievements such as the first measurement of the $W$ boson mass at the LHC, which indeed requires a control of many QCD effects at unprecedented level and skepticism is in order. It is vital that we stay alert to the limitations of the theory.

\section{Acknowledgements}

I would like to thank Stefano Forte, Aharon Levy and Giovanni Ridolfi for taking the initiative for this book in memory and honour of Guido Altarelli, for inviting me to contribute, as well as for giving valuable feedback on the text.

\section{References}

[1] G. Altarelli, Concluding Talk: A Theorist Overview on Particle Physics, in Flavor physics and CP violation. Proceedings, 9th International Conference, FPCP 2011, Maale HaChamisha, Israel, 2011, arXiv:1108.3514.

[2] L. Evans and P. Bryant, LHC Machine, JINST 3 (2008) S08001.

[3] ATLAS Collaboration, The ATLAS Experiment at the CERN Large Hadron Collider, JINST 3 (2008) S08003.

[4] CMS Collaboration, The CMS experiment at the CERN LHC, JINST 3 (2008) S08004.

[5] G. Altarelli, The Higgs: so simple yet so unnatural, Phys. Scripta T158 (2013) 014011 arXiv:1308.0545].

[6] ATLAS collaboration, G. Aad et al., Observation of a new particle in the search for the Standard Model Higgs boson with the ATLAS detector at the LHC, Phys. Lett. B716 (2012) 1 arXiv: 1207.7214.

[7] CMS collaboration, S. Chatrchyan et al., Observation of a new boson at a mass of 125 GeV with the CMS experiment at the LHC, Phys. Lett. B716 (2012) 30 arXiv:1207.7235. 
[8] G. Altarelli, Particle Physics at the LHC Start, Prog. Theor. Phys. Suppl. 187 (2011) 305 arXiv: 1010.5637.

[9] V. N. Gribov and L. N. Lipatov, Deep inelastic ep scattering in perturbation theory, Sov. J. Nucl. Phys. 15 (1972) 438.

[10] V. N. Gribov and L. N. Lipatov, $e^{+} e^{-}$pair annihilation and deep inelastic ep scattering in perturbation theory, Sov. J. Nucl. Phys. 15 (1972) 675.

[11] Y. L. Dokshitzer, Calculation of the Structure Functions for Deep Inelastic Scattering and $e^{+} e^{-}$Annihilation by Perturbation Theory in Quantum Chromodynamics, Sov. Phys. JETP 46 (1977) 641.

[12] G. Altarelli and G. Parisi, Asymptotic Freedom in Parton Language, Nucl. Phys. B126 (1977) 298.

[13] A. Vogt, S. Moch and J. A. M. Vermaseren, The Three-loop splitting functions in QCD: The Singlet case, Nucl. Phys. B691 (2004) 129 arXiv:hep-ph/0404111.

[14] S. Moch, J. A. M. Vermaseren and A. Vogt, The Three loop splitting functions in QCD: The Nonsinglet case, Nucl. Phys. B688 (2004) 101 arXiv:hep-ph/0403192.

[15] G. Altarelli, R. K. Ellis and G. Martinelli, Large Perturbative Corrections to the Drell-Yan Process in QCD, Nucl. Phys. B157 (1979) 461.

[16] S. D. Drell and T.-M. Yan, Massive Lepton Pair Production in Hadron-Hadron Collisions at High-Energies, Phys. Rev. Lett. 25 (1970) 316.

[17] LHCB collaboration, A. A. Alves, Jr. et al., The LHCb Detector at the LHC, JINST 3 (2008) S08005.

[18] ALICE collaboration, K. Aamodt et al., The ALICE experiment at the CERN LHC, JINST 3 (2008) S08002.

[19] ATLAS Collaboration, Summary Plots, 2017, https://atlas.web.cern.ch/Atlas/ GROUPS/PHYSICS/CombinedSummaryPlots/SM/index.html.

[20] CMS Collaboration, Summary Plots, 2017, https://twiki.cern.ch/twiki/bin/view/CMSPublic/PhysicsResultsCombined.

[21] ATLAS collaboration, M. Aaboud et al., Measurement of the prompt J/ $\psi$ pair production cross-section in pp collisions at $\sqrt{s}=8 \mathrm{TeV}$ with the ATLAS detector, Eur. Phys. J. C77 (2017) 76 arXiv:1612.02950].

[22] T. Sjöstrand, S. Mrenna and P. Z. Skands, A Brief Introduction to PYTHIA 8.1, Comput. Phys. Commun. 178 (2008) 852 arXiv:0710.3820.

[23] J. Bellm et al., Herwig 7.0/Herwig + 3.0 release note, Eur. Phys. J. C 76 (2016) 196 arXiv:1512.01178.

[24] T. Pierog, I. Karpenko, J. M. Katzy, E. Yatsenko and K. Werner, EPOS LHC: Test of collective hadronization with data measured at the CERN Large Hadron Collider, Phys. Rev. C92 (2015) 034906 arXiv:1306.0121. 
[25] ALICE collaboration, J. Adam et al., Enhanced production of multi-strange hadrons in high-multiplicity proton-proton collisions, Nature Phys. 13 (2017) 535 arXiv:1606.07424.

[26] CMS collaboration, V. Khachatryan et al., Measurement of the double-differential inclusive jet cross section in proton-proton collisions at $\sqrt{s}=13$ TeV, Eur. Phys. J. C76 (2016) 451 arXiv: 1605.04436.

[27] CMS collaboration, V. Khachatryan et al., Measurement and QCD analysis of double-differential inclusive jet cross sections in pp collisions at $\sqrt{s}=8 \mathrm{TeV}$ and cross section ratios to 2.76 and $7 \mathrm{TeV}$, JHEP 03 (2017) 156 arXiv:1609.05331.

[28] CMS collaboration, A. M. Sirunyan et al., Measurement of the triple-differential dijet cross section in proton-proton collisions at $\sqrt{s}=8 \mathrm{TeV}$ and constraints on parton distribution functions, Eur. Phys. J. C77 (2017) 746 arXiv:1705.02628.

[29] ATLAS collaboration, M. Aaboud et al., Measurement of inclusive jet and dijet cross-sections in proton-proton collisions at $\sqrt{s}=13$ TeV with the ATLAS detector, arXiv:1711.02692.

[30] ATLAS collaboration, M. Aaboud et al., Measurement of the inclusive jet cross-sections in proton-proton collisions at $\sqrt{s}=8 \mathrm{TeV}$ with the ATLAS detector, JHEP 09 (2017) 020 arXiv: 1706.03192.

[31] J. Currie, E. W. N. Glover and J. Pires, Next-to-Next-to Leading Order QCD Predictions for Single Jet Inclusive Production at the LHC, Phys. Rev. Lett. 118 (2017) 072002 arXiv:1611.01460.

[32] J. Currie, E. W. N. Glover, T. Gehrmann, A. Gehrmann-De Ridder, A. Huss and J. Pires, Single Jet Inclusive Production for the Individual Jet $p_{\mathrm{T}}$ Scale Choice at the LHC, Acta Phys. Polon. B48 (2017) 955 arXiv:1704.00923].

[33] LHCB collaboration, R. Aaij et al., Measurements of prompt charm production cross-sections in pp collisions at $\sqrt{s}=13 \mathrm{TeV}$, JHEP 03 (2016) 159 arXiv:1510.01707.

[34] PROSA collaboration, O. Zenaiev et al., Impact of heavy-flavour production cross sections measured by the LHCb experiment on parton distribution functions at low $x$, Eur. Phys. J. C75 (2015) 396 arXiv: 1503.04581].

[35] R. Gauld and J. Rojo, Precision determination of the small-x gluon from charm production at LHCb, Phys. Rev. Lett. 118 (2017) 072001 arXiv:1610.09373.

[36] M. Czakon, D. Heymes and A. Mitov, High-precision differential predictions for top-quark pairs at the LHC, Phys. Rev. Lett. 116 (2016) 082003 arXiv:1511.00549.

[37] CMS collaboration, V. Khachatryan et al., Measurement of the top quark mass using proton-proton data at $\sqrt{s}=7$ and 8 TeV, Phys. Rev. D93 (2016) 072004 arXiv:1509.04044. 
[38] ATLAS Collaboration, Measurement of the top quark mass in the $t \bar{t} \rightarrow$ lepton + jets channel from $\sqrt{s}=8 \mathrm{TeV}$ ATLAS data, 2017, ATLAS-CONF-2017-071, https://cds.cern.ch/record/2285809.

[39] CMS collaboration, V. Khachatryan et al., Measurement of the t-tbar production cross section in the e-mu channel in proton-proton collisions at $\sqrt{s}=7$ and $8 \mathrm{TeV}$, JHEP 08 (2016) 029 arXiv: 1603.02303.

[40] ATLAS collaboration, M. Aaboud et al., Measurement of lepton differential

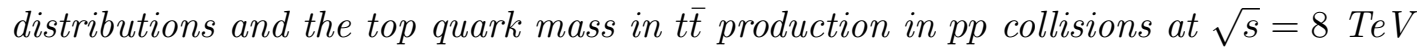
with the ATLAS detector, Eur. Phys. J. C77 (2017) 804 arXiv:1709.09407.

[41] CMS collaboration, V. Khachatryan et al., Measurement of differential cross sections for top quark pair production using the lepton+jets final state in proton-proton collisions at 13 TeV, Phys. Rev. D95 (2017) 092001 arXiv:1610.04191.

[42] G. Altarelli, The QCD Running Coupling and its Measurement, PoS Corfu2012 (2013) 002 arXiv:1303.6065.

[43] CMS collaboration, S. Chatrchyan et al., Determination of the top-quark pole mass and strong coupling constant from the $t \bar{t}$ production cross section in pp collisions at $\sqrt{s}=7 \mathrm{TeV}$, Phys. Lett. B728 (2014) 496 arXiv:1307.1907.

[44] T. Klijnsma, S. Bethke, G. Dissertori and G. P. Salam, Determination of the strong coupling constant $\alpha_{s}\left(m_{Z}\right)$ from measurements of the total cross section for top-antitop quark production, Eur. Phys. J. C77 (2017) 778 arXiv:1708.07495.

[45] UA1 collaboration, G. Arnison et al., Experimental Observation of Isolated Large Transverse Energy Electrons with Associated Missing Energy at $s^{1 / 2}=540 \mathrm{GeV}$, Phys. Lett. 122B (1983) 103.

[46] UA2 collaboration, M. Banner et al., Observation of Single Isolated Electrons of High Transverse Momentum in Events with Missing Transverse Energy at the CERN $\bar{p} p$ Collider, Phys. Lett. 122B (1983) 476.

[47] UA1 collaboration, G. Arnison et al., Experimental Observation of Lepton Pairs of Invariant Mass Around $95 \mathrm{GeV} / \mathrm{c}^{2}$ at the CERN SPS Collider, Phys. Lett. 126B (1983) 398.

[48] UA2 collaboration, P. Bagnaia et al., Evidence for $Z^{0} \rightarrow e^{+} e^{-}$at the CERN $\bar{p} p$ Collider, Phys. Lett. 129B (1983) 130.

[49] J. C. Collins, D. E. Soper and G. F. Sterman, All Order Factorization for Drell-Yan Cross-sections, Phys. Lett. 134B (1984) 263

[50] G. T. Bodwin, Factorization of the Drell-Yan Cross-Section in Perturbation Theory, Phys. Rev. D31 (1985) 2616.

[51] G. Altarelli, The Early Days of QCD (as seen from Rome), Nuovo Cim. C035N1 (2012) 1 arXiv:1106.3189. 
[52] G. Altarelli, R. K. Ellis and G. Martinelli, Leptoproduction and Drell-Yan Processes Beyond the Leading Approximation in Chromodynamics, Nucl. Phys. B143 (1978) 521

[53] J. Kubar-Andre and F. E. Paige, Gluon Corrections to the Drell-Yan Model, Phys. Rev. D19 (1979) 221.

[54] J. Kubar, M. Le Bellac, J. L. Meunier and G. Plaut, QCD Corrections to the Drell-Yan Mechanism and the Pion Structure Function, Nucl. Phys. B175 (1980) 251.

[55] R. Hamberg, W. L. van Neerven and T. Matsuura, A complete calculation of the order $\alpha_{s}^{2}$ correction to the Drell-Yan K factor, Nucl. Phys. B359 (1991) 343.

[56] W. L. van Neerven and E. B. Zijlstra, The $O\left(\alpha_{s}^{2}\right)$ corrected Drell-Yan K factor in the $D I S$ and $M S$ scheme, Nucl. Phys. B382 (1992) 11.

[57] P. J. Rijken and W. L. van Neerven, Order $\alpha_{s}^{2}$ contributions to the Drell-Yan cross-section at fixed target energies, Phys. Rev. D51 (1995) 44 arXiv:hep-ph/9408366.

[58] C. Anastasiou, L. J. Dixon, K. Melnikov and F. Petriello, High precision QCD at hadron colliders: Electroweak gauge boson rapidity distributions at NNLO, Phys. Rev. D69 (2004) 094008 arXiv: hep-ph/0312266.

[59] K. Melnikov and F. Petriello, Electroweak gauge boson production at hadron colliders through $O\left(\alpha_{s}^{2}\right)$, Phys. Rev. D74 (2006) 114017 arXiv:hep-ph/0609070].

[60] S. Catani, L. Cieri, G. Ferrera, D. de Florian and M. Grazzini, Vector boson production at hadron colliders: a fully exclusive QCD calculation at NNLO, Phys. Rev. Lett. 103 (2009) 082001 arXiv:0903.2120.

[61] R. Gavin, Y. Li, F. Petriello and S. Quackenbush, FEWZ 2.0: A code for hadronic Z production at next-to-next-to-leading order, Comput. Phys. Commun. 182 (2011) 2388 arXiv:1011.3540.

[62] J. M. Lindert et al., Precise predictions for $V+$ jets dark matter backgrounds, Eur. Phys. J. C77 (2017) 829 arXiv:1705.04664.

[63] ATLAS collaboration, M. Aaboud et al., Precision measurement and interpretation of inclusive $W^{+}, W^{-}$and $Z / \gamma^{*}$ production cross sections with the ATLAS detector, Eur. Phys. J. C77 (2017) 367 arXiv:1612.03016.

[64] ATLAS collaboration, G. Aad et al., Measurement of the double-differential high-mass Drell-Yan cross section in pp collisions at $\sqrt{s}=8 \mathrm{TeV}$ with the ATLAS detector, JHEP 08 (2016) 009 arXiv: 1606.01736.

[65] CMS collaboration, V. Khachatryan et al., Measurements of differential and double-differential Drell-Yan cross sections in proton-proton collisions at $8 \mathrm{TeV}$, Eur. Phys. J. C75 (2015) 147 arXiv:1412.1115.

[66] CMS collaboration, V. Khachatryan et al., Measurement of the differential cross section and charge asymmetry for inclusive $\mathrm{pp} \rightarrow \mathrm{W}^{ \pm}+X$ production at $\sqrt{s}=8$ TeV, Eur. Phys. J. C76 (2016) 469 arXiv: 1603.01803. 
[67] LHCB collaboration, R. Aaij et al., Measurement of forward $W$ and $Z$ boson production in pp collisions at $\sqrt{s}=8 \mathrm{TeV}$, JHEP 01 (2016) 155 arXiv:1511.08039.

[68] LHCB collaboration, R. Aaij et al., Measurement of the forward $Z$ boson production cross-section in pp collisions at $\sqrt{s}=13 \mathrm{TeV}$, JHEP 09 (2016) 136 arXiv:1607.06495.

[69] ZEUS, H1 collaboration, H. Abramowicz et al., Combination of measurements of inclusive deep inelastic $e^{ \pm} p$ scattering cross sections and QCD analysis of HERA data, Eur. Phys. J. C75 (2015) 580 arXiv: 1506.06042.

[70] L. A. Harland-Lang, A. D. Martin, P. Motylinski and R. S. Thorne, Parton distributions in the LHC era: MMHT 2014 PDFs, Eur. Phys. J. C75 (2015) 204 arXiv:1412.3989.

[71] S. Dulat et al., New parton distribution functions from a global analysis of quantum chromodynamics, Phys. Rev. D93 (2016) 033006 arXiv: 1506.07443.

[72] S. Alekhin, J. Blümlein, S. Moch and R. Placakyte, Parton distribution functions, $\alpha_{s}$, and heavy-quark masses for LHC Run II, Phys. Rev. D96 (2017) 014011 arXiv:1701.05838.

[73] NNPDF collaboration, R. D. Ball et al., Parton distributions from high-precision collider data, Eur. Phys. J. C77 (2017) 663 arXiv:1706.00428.

[74] S. Alekhin, J. Blümlein and S. Moch, The ABM parton distributions tuned to LHC data, Phys. Rev. D89 (2014) 054028 arXiv:1310.3059.

[75] NNPDF collaboration, R. D. Ball et al., Parton distributions for the LHC Run II, JHEP 04 (2015) 040 arXiv: 1410.8849.

[76] ATLAS collaboration, G. Aad et al., Measurement of the inclusive $W^{ \pm}$and $Z / \gamma^{*}$ cross sections in the electron and muon decay channels in pp collisions at $\sqrt{s}=7 \mathrm{TeV}$ with the ATLAS detector, Phys. Rev. D85 (2012) 072004 arXiv:1109.5141.

[77] ATLAS collaboration, G. Aad et al., Measurement of the transverse momentum and $\phi_{\eta}^{*}$ distributions of Drell-Yan lepton pairs in proton-proton collisions at $\sqrt{s}=8 \mathrm{TeV}$ with the ATLAS detector, Eur. Phys. J. C76 (2016) 291 arXiv:1512.02192.

[78] CMS collaboration, V. Khachatryan et al., Measurement of the $Z$ boson differential cross section in transverse momentum and rapidity in protonproton collisions at 8 TeV, Phys. Lett. B749 (2015) 187 [arXiv:1504.03511.

[79] CMS collaboration, V. Khachatryan et al., Measurement of the transverse momentum spectra of weak vector bosons produced in proton-proton collisions at $\sqrt{s}=8 \mathrm{TeV}$, JHEP 02 (2017) 096 arXiv: 1606.05864].

[80] A. Gehrmann-De Ridder, T. Gehrmann, E. W. N. Glover, A. Huss and T. A. Morgan, Precise $Q C D$ predictions for the production of a $Z$ boson in association with a hadronic jet, Phys. Rev. Lett. 117 (2016) 022001 arXiv:1507.02850]. 
[81] R. Boughezal, J. M. Campbell, R. K. Ellis, C. Focke, W. T. Giele, X. Liu et al., Z-boson production in association with a jet at next-to-next-to-leading order in perturbative QCD, Phys. Rev. Lett. 116 (2016) 152001 arXiv: 1512.01291.

[82] R. Boughezal, A. Guffanti, F. Petriello and M. Ubiali, The impact of the LHC Z-boson transverse momentum data on PDF determinations, JHEP 07 (2017) 130 arXiv:1705.00343.

[83] A. Gehrmann-De Ridder, T. Gehrmann, E. W. N. Glover, A. Huss and T. A. Morgan, The NNLO QCD corrections to $Z$ boson production at large transverse momentum, JHEP 07 (2016) 133 arXiv: 1605.04295.

[84] GFITTER collaboration, M. Baak et al., The global electroweak fit at NNLO and prospects for the LHC and ILC, Eur. Phys. J. C74 (2014) 3046 arXiv:1407.3792.

[85] J. de Blas et al., Electroweak precision observables and Higgs-boson signal strengths in the Standard Model and beyond: present and future, JHEP 12 (2016) 135 arXiv:1608.01509.

[86] S. Heinemeyer, W. Hollik, G. Weiglein and L. Zeune, Implications of LHC search results on the $W$ boson mass prediction in the MSSM, JHEP 12 (2013) 084 arXiv:1311.1663.

[87] G. Altarelli, March, 1998, LHCC meeting.

[88] ATLAS collaboration, M. Aaboud et al., Measurement of the $W$-boson mass in pp collisions at $\sqrt{s}=7$ TeV with the ATLAS detector, Eur. Phys. J. C78 (2018) 110 arXiv:1701.07240.

[89] CMS Collaboration, Measurement of the weak mixing angle with the forward-backward asymmetry of Drell-Yan events at 8 TeV, 2017, https://cds.cern.ch/record/2273392.

[90] M. J. G. Veltman, Second Threshold in Weak Interactions, Acta Phys. Polon. B8 (1977) 475.

[91] B. W. Lee, C. Quigg and H. B. Thacker, The Strength of Weak Interactions at Very High-Energies and the Higgs Boson Mass, Phys. Rev. Lett. 38 (1977) 883.

[92] B. W. Lee, C. Quigg and H. B. Thacker, Weak Interactions at Very High-Energies: The Role of the Higgs Boson Mass, Phys. Rev. D16 (1977) 1519.

[93] ATLAS collaboration, G. Aad et al., Measurements of $W \gamma$ and $Z \gamma$ production in pp collisions at $\sqrt{s}=7 T e V$ with the ATLAS detector at the LHC, Phys. Rev. D87 (2013) 112003 arXiv:1302.1283.

[94] M. Grazzini, S. Kallweit and D. Rathlev, $W \gamma$ and $Z \gamma$ production at the LHC in NNLO QCD, JHEP 07 (2015) 085 arXiv:1504.01330.

[95] M. Grazzini, S. Kallweit, D. Rathlev and M. Wiesemann, $W^{ \pm} Z$ production at the LHC: fiducial cross sections and distributions in NNLO QCD, JHEP 05 (2017) 139 arXiv:1703.09065. 
[96] F. Caola, K. Melnikov, R. Röntsch and L. Tancredi, QCD corrections to ZZ production in gluon fusion at the LHC, Phys. Rev. D92 (2015) 094028 [arXiv: 1509.06734].

[97] ATLAS collaboration, G. Aad et al., Measurements of four-lepton production in pp collisions at $\sqrt{s}=8 \mathrm{TeV}$ with the ATLAS detector, Phys. Lett. B753 (2016) 552 arXiv:1509.07844.

[98] CMS collaboration, A. M. Sirunyan et al., Measurements of the $\mathrm{pp} \rightarrow$ ZZ production cross section and the $\mathrm{Z} \rightarrow 4 \ell$ branching fraction, and constraints on anomalous triple gauge couplings at $\sqrt{s}=13$ TeV, Eur. Phys. J. C78 (2018) 165 arXiv:1709.08601.

[99] F. Caola and K. Melnikov, Constraining the Higgs boson width with ZZ production at the LHC, Phys. Rev. D88 (2013) 054024 arXiv: 1307.4935.

[100] ATLAS collaboration, G. Aad et al., Evidence of $W \gamma \gamma$ Production in pp Collisions at $\sqrt{s}=8 \mathrm{TeV}$ and Limits on Anomalous Quartic Gauge Couplings with the ATLAS Detector, Phys. Rev. Lett. 115 (2015) 031802 [arXiv: 1503.03243].

[101] ATLAS collaboration, G. Aad et al., Measurements of $Z \gamma$ and $Z \gamma \gamma$ production in $p p$ collisions at $\sqrt{s}=8 \mathrm{TeV}$ with the ATLAS detector, Phys. Rev. D93 (2016) 112002 arXiv:1604.05232.

[102] CMS collaboration, A. M. Sirunyan et al., Measurements of the $p p \rightarrow W \gamma \gamma$ and $p p$ $\rightarrow Z \gamma \gamma$ cross sections and limits on anomalous quartic gauge couplings at $\sqrt{s}=8$ $T e V$, JHEP 10 (2017) 072 [arXiv: 1704.00366].

[103] J. D. Bjorken, Rapidity gaps and jets as a new physics signature in very high-energy hadron hadron collisions, Phys. Rev. D47 (1993) 101.

[104] CMS collaboration, V. Khachatryan et al., Measurement of electroweak production of two jets in association with a $Z$ boson in proton-proton collisions at $\sqrt{s}=8 \mathrm{TeV}$, Eur. Phys. J. C75 (2015) 66 arXiv:1410.3153.

[105] CMS collaboration, V. Khachatryan et al., Measurement of electroweak production of $a W$ boson and two forward jets in proton-proton collisions at $\sqrt{s}=8 \mathrm{TeV}$, JHEP 11 (2016) 147 arXiv:1607.06975.

[106] ATLAS collaboration, G. Aad et al., Measurement of the electroweak production of dijets in association with a Z-boson and distributions sensitive to vector boson fusion in proton-proton collisions at $\sqrt{s}=8 \mathrm{TeV}$ using the ATLAS detector, JHEP 04 (2014) 031 arXiv:1401.7610.

[107] ATLAS collaboration, M. Aaboud et al., Measurements of electroweak Wjj production and constraints on anomalous gauge couplings with the ATLAS detector, Eur. Phys. J. C77 (2017) 474 arXiv:1703.04362].

[108] ATLAS collaboration, M. Aaboud et al., Measurement of the cross-section for electroweak production of dijets in association with a $Z$ boson in pp collisions at $\sqrt{s}=$ $13 \mathrm{TeV}$ with the ATLAS detector, Phys. Lett. B775 (2017) 206 [arXiv:1709.10264]. 
[109] CMS collaboration, A. M. Sirunyan et al., Observation of electroweak production of same-sign $W$ boson pairs in the two jet and two same-sign lepton final state in proton-proton collisions at $\sqrt{s}=13$ TeV, Phys. Rev. Lett. 120 (2018) 081801 arXiv:1709.05822.

[110] ATLAS, CMS collaboration, G. Aad et al., Combined Measurement of the Higgs Boson Mass in pp Collisions at $\sqrt{s}=7$ and 8 TeV with the ATLAS and CMS Experiments, Phys. Rev. Lett. 114 (2015) 191803 arXiv:1503.07589.

[111] ATLAS, CMS collaboration, G. Aad et al., Measurements of the Higgs boson production and decay rates and constraints on its couplings from a combined ATLAS and CMS analysis of the LHC pp collision data at $\sqrt{s}=7$ and 8 TeV, JHEP 08 (2016) 045 arXiv: 1606.02266.

[112] CMS collaboration, V. Khachatryan et al., Constraints on the spin-parity and anomalous HVV couplings of the Higgs boson in proton collisions at 7 and $8 \mathrm{TeV}$, Phys. Rev. D92 (2015) 012004 [arXiv: 1411.3441].

[113] ATLAS collaboration, G. Aad et al., Study of the spin and parity of the Higgs boson in diboson decays with the ATLAS detector, Eur. Phys. J. C75 (2015) 476 arXiv:1506.05669.

[114] ATLAS Collaboration, Combined measurements of Higgs boson production and decay in the $H \rightarrow Z Z^{*} \rightarrow 4 \ell$ and $H \rightarrow \gamma \gamma$ channels using $\sqrt{s}=13$ TeV proton-proton collision data collected with the ATLAS experiment, 2017, ATLAS-CONF-2017-047, https://cds.cern.ch/record/2273854.

[115] ATLAS collaboration, M. Aaboud et al., Evidence for the $H \rightarrow b \bar{b}$ decay with the ATLAS detector, JHEP 12 (2017) 024 arXiv:1708.03299.

[116] CMS collaboration, A. M. Sirunyan et al., Evidence for the Higgs boson decay to a bottom quark-antiquark pair, Phys. Lett. B780 (2018) 501 arXiv:1709.07497.

[117] ATLAS collaboration, M. Aaboud et al., Evidence for the associated production of the Higgs boson and a top quark pair with the ATLAS detector, arXiv:1712.08891.

[118] CMS collaboration, A. M. Sirunyan et al., Evidence for associated production of a Higgs boson with a top quark pair in final states with electrons, muons, and hadronically decaying $\tau$ leptons at $\sqrt{s}=13 \mathrm{TeV}$, arXiv:1803.05485.

[119] C. Anastasiou, C. Duhr, F. Dulat, F. Herzog and B. Mistlberger, Higgs Boson Gluon-Fusion Production in QCD at Three Loops, Phys. Rev. Lett. 114 (2015) 212001 arXiv:1503.06056. 